\section{Heller, Johann Florian}

\author{
A. M. Gressner ${ }^{1}$ und O. A. Gressner ${ }^{2}$ \\ ${ }^{1}$ Labor Dr. Wisplinghoff Berlin, Berlin, Deutschland \\ ${ }^{2}$ Labor Dr. Wisplinghoff Köln, Köln, Deutschland
}

Lebensdaten Tschechischer Chemiker, geboren am 4. Mai 1813 in Iglau (Tschechien), gestorben am 21. November 1871 in Wien.

Verdienste Heller erhielt bereits während seiner Gymnasialzeit Unterricht in praktischer Chemie von seinem als Apotheker tätigen Vater, studierte Chemie in Prag, schloss mit einem pharmazeutischen Examen ab und promovierte im Jahr 1837 in Prag zum Dr. chem. Neben dem Studium der Chemie belegte Heller medizinische Vorlesungen und Kurse. Nach Übersiedlung nach Wien im Jahr 1838 widmete er sich dem Studium pathochemischer Prozesse und veranstaltete chemische Kurse für Medizinstudenten und private Kurse für Ärzte in der physiologischen und pathologischen Chemie und Mikroskopie. 1844 übernahm Heller die Leitung des Pathologisch-Chemischen Laboratoriums am Wiener Allgemeinen Krankenhaus, wurde aber erst 1855 zum offiziellen Leiter ernannt. Seine herausragenden Beiträge zur Klinischen Chemie ( $\triangleright$ Klinische Chemie) betreffen die programmatische
Definition der Aufgaben der Klinischen Chemie, die Entwicklung neuer Testmethoden (z. B. Heller-Ringprobe), die systematische chemische Analyse von Körperflüssigkeiten in definierten Krankheitszuständen, die medizinische Interpretation klinisch-chemischer Befunde, die Organisation des Pathologisch-Chemischen Laboratoriums am Wiener Allgemeinen Krankenhaus sowie die Tätigkeit als Editor des ersten Journals der Klinischen Chemie („Hellers Archiv für physiologische und pathologische Chemie und Mikroskopie“, das in 6 Bänden zwischen 1844 und 1853 erschien). Heller betonte und entwickelte die pathologische Chemie als eine unverzichtbare adjuvante Disziplin für eine wissenschaftlich orientierte Medizin. Zusammen mit Scherer $(\triangleright$ Scherer, Johann Joseph von) und Simon ( $\triangleright$ Simon, Johann Franz) gehört er zu den Begründern der Klinischen Chemie.

\section{Literatur}

Schmalhofer J (1980) Das Werk von Johann Florian Heller mit besonderer Berücksichtigung der Entstehung des ersten pathologischchemischen Laboratoriums im Allgemeinen Krankenhaus und der Ernennung Hellers zum Vorsitzenden des Laboratoriums. Dissertation, Bonn 